\title{
Quantification of amyloid deposition using bone scan agents
}

\author{
Takashi Kudo, MD, PhD, ${ }^{\mathrm{a}, \mathrm{b}}$ and Aiganym Imakhanova, $\mathrm{MD}^{\mathrm{b}}$ \\ a Department of Radioisotope Medicine, Atomic Bomb Disease Institute, Nagasaki University, \\ Nagasaki, Japan \\ b Department of Radioisotope Medicine, Nagasaki University Graduate School of Biomedical \\ Sciences, Nagasaki, Japan
}

Received Aug 7, 2020; accepted Aug 7, 2020

doi: 10.1007/s12350-020-02340-9

\section{See related article, pp. 506-514}

\section{CARDIAC AMYLOIDOSIS: EMERGING REQUIREMENTS FOR AN ACCURATE DIAGNOSIS}

Cardiac amyloidosis is one of the most underdiagnosed cardiovascular disease entities. It was previously considered to be a rare condition, but was recently identified as one of the main causes of heart failure, particularly HFpHF (heart failure with preserved ejection fraction), in elderly patients. ${ }^{1}$ There are several subtypes of amyloid, with major cardiac effects being AL (light-chain) and ATTR (transthyretin) amyloidosis. $\mathrm{AL}$ amyloidosis occurs as a consequence of immunoglobulin-related diseases, such as multiple myeloma, and has a very poor prognosis. ATTR amyloidosis is subdivided into wild and mutant types. The mutant type occurs infrequently with genetic changes and a clear family history. In contrast, wild-type ATTR amyloidosis, also known as senile systemic amyloidosis (SSA), is caused by the deposition of wild-type ATTR amyloid on multiple organs, including the myocardium, and is common in elderly patients. A population-based study conducted in Finland ${ }^{2}$ revealed a strikingly high prevalence of the deposition of ATTR in very old (over $85)$ subjects $(25 \%)$.

The difficulties associated with diagnosing cardiac amyloidosis have resulted in its underdiagnosis. The

\footnotetext{
Reprint requests: Takashi Kudo, MD, PhD, Department of Radioisotope Medicine, Atomic Bomb Disease Institute, Nagasaki University, Nagasaki, Japan; tkudo123@nagasaki-u.ac.jp J Nucl Cardiol 2022;29:515-8.

$1071-3581 / \$ 34.00$

Copyright (C) 2020 American Society of Nuclear Cardiology.
}

classical "stigmata" symptoms of systemic amyloidosis include "panda eyes", macroglossia, carpal tunnel symptoms, and nail dystrophy. However, patients with ATTR amyloidosis may exhibit few of the characteristic symptoms and signs of this disease, which may be overlooked when ATTR amyloidosis is not one of the diagnostic options in the attending clinician's mind. However, the importance of this disease is now being recognized, and many cardiologists consider cardiac amyloidosis as one of the causes of unexplained heart failure in the elderly. Since invasive biopsy is required for the final diagnosis of cardiac amyloidosis, the development of non-invasive diagnostic procedures is needed.

Several non-invasive methods, such as apical sparing in echocardiography ${ }^{3}$ and $\mathrm{T} 1$ mapping in $\mathrm{CMR},{ }^{4}$ have good diagnostic accuracy. However, the findings of these methods are not directly related to the molecular etiology of cardiac amyloidosis. The background of nuclear cardiology imaging involves molecular imaging and provides a direct link between the findings obtained and etiology. Three nuclear medicine imaging techniques are performed in clinical settings: planar, SPECT, and PET. Several tracers are now available for amyloid PET imaging, including C-11 PIB (Pittsburgh compound-B) ${ }^{5}$ and $\mathrm{F}-18$-labeled tracers (florbetapir, ${ }^{6}$ flutemetamol, ${ }^{7}$ and florbetaben ${ }^{8}$ ), which were originally developed for amyloid imaging in the brain. Amyloid PET imaging is a well-established technique in neuroscience, and its usefulness for cardiac amyloidosis has also been demonstrated. ${ }^{9}$ PET tracers require specific synthetic techniques and are expensive. However, there are currently no alternatives for detecting amyloid deposition in the brain. On the other hand, we have good old alternatives for cardiac imaging. Tc-99mlabeled pyrophosphate agents for bone imaging, such as Tc-99m PYP, Tc-99m HMDP, and Tc-99m DPD, referred to as bone scan agents, are used to detect the 
myocardial deposition of amyloid. (Another agent, Tc99m MDP has also been used, but was found to provide less accurate findings in cardiac amyloidosis imaging than other bone scan agents. ${ }^{10}$ )

The imaging of ATTR cardiac amyloidosis using bone scan agents allows for the detection of cardiac amyloidosis and easily differentiates ATTR amyloidosis from AL amyloidosis with simple conventional planar imaging. ${ }^{11}$ Therefore, it is now employed in disease detection and differential diagnoses.

\section{WHY ARE MORE PRECISE MEASUREMENTS NEEDED?}

A recently proposed method to assess the myocardial uptake of bone scan agents uses planar frontal view images. ${ }^{11}$ It is a very simple method that compares heart and contralateral lung activities (heart to contralateral lung ratio: $\mathrm{H} / \mathrm{CL}$ ) using planar images and provides reliable findings. The first study to use this technique for ATTR amyloidosis reported very high sensitivity and specificity with only one false negative. However, sensitivity and specificity both decreased as more clinical researchers performed this method. A multicenter study reported sensitivity and specificity of $91 \%$ and $92 \%$, respectively, ${ }^{12}$ which are very high, but further technical improvements are needed.

Measurements using planar images have several intrinsic limitations. Planar images obtained from the frontal view include photons from the posterior side of the body. In the case of the chest, planar images show rib uptake. The frontal view includes faint photons from ribs on the posterior side of the body, which is referred to as "shine-through". It mainly overlaps on the contralateral lung ROI because the mass of the heart shields photons from the posterior ribs, which has a negative impact on quantitative measurements. Furthermore, rib cartilage sometimes shows the significant uptake of bone scan agents, which overlap on the heart and contralateral lung. This results in errors in the measurement of myocardial uptake. Increases in soft tissue uptake with amyloid deposition, as reported in the article by Bellevre et al., ${ }^{13}$ may also contribute to measurement errors.

However, quantitative measurements using the SPECT technique are more challenging than those using PET. In PET imaging using F-18 amyloid tracers, SUV values are useful for quantitative measurements. ${ }^{9}$ However, due to the poor image quality of SPECT, the quantification of myocardial tracer concentrations is not reliable. Recent advances in imaging techniques, including solid-state detectors, will improve the reliability of findings obtained using SPECT.
In this issue of the Journal of Nuclear Cardiology, Bellevre et al. quantified amyloid deposition in patients using the bone scan agent, Tc-99m HMDP with a CZT solid-state detector gamma camera. ${ }^{13}$ The CZT camera has a number of advantages over the conventional Anger-type gamma camera, particularly in the field of cardiology. Amyloid bone scan agents imaging has already been attempted using CZT camera. ${ }^{14,15}$ However, the majority of these studies used the H/CL method originally introduced by Bokuhari. ${ }^{11}$ The value of SUV, which was used in the present study, was originally developed for use in FDG PET in oncology. In this issue of JNC, Bellevre et al. demonstrated that SUV values obtained using the CZT camera were sufficiently reliable for clinical use. The findings obtained revealed high sensitivity and specificity of $100 \%$ using the quantitative SUVmax method for diagnosis as well as the absence of the large overlap observed in the H/CL method. These findings indicate the superiority of quantitative methods over semi-quantitative methods, such as ratio measurements.

In the field of oncology, a number of "daughter', parameters are derived from SUV (Table 1), and those are still under debate as to which are the best. We can start a similar debate searching for daughter parameters that are suitable for specific purpose of imaging. The present study used two methods to quantify myocardial tracer uptake. The SUVmax method was used to calculate quantitative uptake normalized to the injected dose and subject body mass, which has been established in PET imaging. The other method was \% ID (injected dose), which is a quantitative method that normalizes tissue uptake to the injected dose. \%ID showed slightly better separation between the control and ATTR groups than SUVmax. In the field of oncology, TLG, which is the sum of FDG uptake in a tumor, is a promising parameter for stratifying the prognosis of cancer patients. By quantifying the total amount of bone tracer deposited in the myocardium, it may be possible to classify the severity of disease in patients. From now on, we can start such debate based on promising results from Bellevre et al.

\section{FUTURE PERSPECTIVES}

Many issues warrant further investigation, such as identifying the most suitable bone scan agents, the best timing to image ATTR cardiac amyloidosis patients, and the most appropriate parameters for prognostication, namely, SUV or \%ID? measuring the sum? Since many advances are occurring in this field, the findings obtained need to be standardized.

A multicenter study performed in the US demonstrated the potential of estimating the prognosis of 
Table 1. A list of quantitative parameters used in FDG (and non-FDG) PET in the field of oncology

\begin{tabular}{|c|c|c|}
\hline Parameter & Calculation & Benefits \\
\hline SUVmax & Maximum pixel value of the lesion & Simple \\
\hline SuVpeak & $\begin{array}{l}\text { Average SUV around pixels } \\
\text { showing SUVmax (Typically a } 1 \text { - } \\
\text { cubic cm sphere) }\end{array}$ & $\begin{array}{l}\text { Eliminates inter-study/scanner variability } \\
\text { caused by different signal to noise } \\
\text { ratios }\end{array}$ \\
\hline SUL & $\begin{array}{l}\text { Similar to SUV, but normalization } \\
\text { is performed with lean body } \\
\text { mass instead of body weight }\end{array}$ & $\begin{array}{l}\text { Minimizes inter/intra-individual } \\
\text { differences caused by different body } \\
\text { consumptions }\end{array}$ \\
\hline TBR (Target to background ratio) & $\begin{array}{l}\text { Ratio between lesions (target) to } \\
\text { normal reference tissue } \\
\text { (Background) }\end{array}$ & $\begin{array}{l}\text { Minimizes inter/intra-individual } \\
\text { differences caused by different } \\
\text { metabolic conditions }\end{array}$ \\
\hline TLG (Total lesion glycolysis) & $\begin{array}{l}\text { Sum of the activity of the lesion } \\
\text { showing high tracer uptake }\end{array}$ & $\begin{array}{l}\text { The activity and volume of the lesion are } \\
\text { both incorporated }\end{array}$ \\
\hline MTV (Metabolic tumor volume) & $\begin{array}{l}\text { Volume of the lesion showing high } \\
\text { tracer uptake }\end{array}$ & $\begin{array}{l}\text { Only measures the volume that is } \\
\text { physiologically active. Available in } \\
\text { accordance with the calculation of TLG }\end{array}$ \\
\hline
\end{tabular}

patients with bone scan agents. Patients with $\mathrm{H} / \mathrm{CL}>$ 1.6 had a poorer prognosis than those with $\mathrm{H} / \mathrm{CL}<$ 1.6. ${ }^{12}$ However, due to the commonly accepted threshold $(\mathrm{H} / \mathrm{CL}=1.5)$, patients with $\mathrm{H} / \mathrm{CL}<1.6$ only account for a small proportion of this patient population. Another study attempted to classify the prognosis of patients using a visual score (Perugini grading scale ${ }^{16}$ ); however, a difference was only observed between grade 0 and another grade. ${ }^{17}$ These findings suggest that the simple planar imaging method is not sufficient to classify the prognosis of patients into multiple grades. Therefore, a stricter and more quantitative method is needed for risk stratification. The present study by Bellevre et al. demonstrated the potential of accurate measurements of amyloid deposition using advanced CZT camera.

So, what must be done in further study using quantitative measurements. First, the selection of suitable patients for expensive therapy should be important. A recent study demonstrated the efficacy of tafamidis, a transthyretin stabilizer, for patients with ATTR amyloidosis. ${ }^{18}$ However, due to the very high prevalence of myocardial amyloid deposition in very old patients, ${ }^{2}$ it is unrealistic to perform tafamidis therapy, which is expensive, for all patients with myocardial amyloid deposition. Therefore, further studies are needed to clarify the magnitude of amyloid deposition in the myocardium that may benefit the most from molecular therapy, such as tafamidis. Is measuring the amount of amyloid deposition on SPECT helpful for the selection of suitable patients for this therapy? Second, the prognosis of patients also needs to be estimated. It currently remains unclear whether the severity of cardiac amyloidosis may be classified using bone scan agent SPECT, similar to FDG PET in the field of oncology. A previous study using F-18 florbetapir reported that the myocardial retention of this tracer significantly differed not only between patients with and without cardiac amyloidosis, but also between those with ATTR and AL amyloidosis. ${ }^{19}$ AL amyloidosis showed significantly higher tracer deposition than ATTR amyloidosis. If this indicates differences in amount of amyloid deposition and a relation to the poorer prognosis of AL amyloidosis than ATTR amyloidosis, measuring the amount of amyloid deposition using bone scan agents may contribute to more accurate estimations of the prognosis of patients. Third, the noninvasive monitoring of therapeutic responses will become more important in near future. In FDG PET in the field of oncology, reductions in the uptake of FDG by tumors generally precede decreases in tumor volumes, and are very useful for separating responders from non-responders in the very early stages of drug therapy. If the use of bone scan agents in the early stages of therapeutic interventions can separate responders from non-responders, it will not only be beneficial for patients, but will also have a positive impact on the associated economic burden.

However, many cardiac amyloidosis patients are still not accurately diagnosed and will continue to be misdiagnosed until it is considered as a common diagnostic option by clinicians. Once cardiac amyloidosis is suspected, nuclear cardiology represents a good, 
reliable, and patient-friendly tool for its diagnosis, as reported by Bellevre et al. ${ }^{13}$

\section{References}

1. Gonzalez-Lopez E, Gallego-Delgado M, Guzzo-Merello G, de Haro-Del Moral FJ, Cobo-Marcos M, Robles C, et al. Wild-type transthyretin amyloidosis as a cause of heart failure with preserved ejection fraction. Eur Heart J. 2015;36:2585-94.

2. Tanskanen M, Peuralinna T, Polvikoski T, Notkola IL, Sulkava R, Hardy J, et al. Senile systemic amyloidosis affects $25 \%$ of the very aged and associates with genetic variation in alpha2-macroglobulin and tau: A population-based autopsy study. Ann Med. 2008;40:232-9.

3. Pagourelias ED, Mirea O, Duchenne J, Van Cleemput J, Delforge $\mathrm{M}$, Bogaert J, et al. Echo parameters for differential diagnosis in cardiac amyloidosis: A head-to-head comparison of deformation and nondeformation parameters. Circ Cardiovasc Imaging. 2017;10:e05588.

4. Martinez-Naharro A, Treibel TA, Abdel-Gadir A, Bulluck H, Zumbo G, Knight DS, et al. Magnetic resonance in transthyretin cardiac amyloidosis. J Am Coll Cardiol. 2017;70:466-77.

5. Klunk WE, Engler H, Nordberg A, Wang Y, Blomqvist G, Holt DP, et al. Imaging brain amyloid in Alzheimer's disease with Pittsburgh Compound-B. Ann Neurol. 2004;55:306-19.

6. Wong DF, Rosenberg PB, Zhou Y, Kumar A, Raymont V, Ravert $\mathrm{HT}$, et al. In vivo imaging of amyloid deposition in Alzheimer disease using the radioligand 18F-AV-45 (florbetapir [corrected] $\mathrm{F}$ 18). J Nucl Med. 2010;51:913-20.

7. Vandenberghe R, Van Laere K, Ivanoiu A, Salmon E, Bastin C, Triau E, et al. 18F-flutemetamol amyloid imaging in Alzheimer disease and mild cognitive impairment: A phase 2 trial. Ann Neurol. 2010;68:319-29.

8. Barthel H, Gertz HJ, Dresel S, Peters O, Bartenstein P, Buerger K, et al. Cerebral amyloid-beta PET with florbetaben $(18 \mathrm{~F})$ in patients with Alzheimer's disease and healthy controls: A multicentre phase 2 diagnostic study. Lancet Neurol. 2011;10:424-35.

9. Dorbala S, Vangala D, Bruyere J Jr, Quarta C, Kruger J, Padera R, et al. Coronary microvascular dysfunction is related to abnormalities in myocardial structure and function in cardiac amyloidosis. JACC Heart Fail. 2014;2:358-67.

10. Lee VW, Caldarone AG, Falk RH, Rubinow A, Cohen AS. Amyloidosis of heart and liver: Comparison of Tc-99m pyrophosphate and Tc-99m methylene diphosphonate for detection. Radiology. 1983;148:239-42.

11. Bokhari S, Castano A, Pozniakoff T, Deslisle S, Latif F, Maurer MS. (99m)Tc-pyrophosphate scintigraphy for differentiating lightchain cardiac amyloidosis from the transthyretin-related familial and senile cardiac amyloidoses. Circ Cardiovasc Imaging. 2013;6:195-201.

12. Castano A, Haq M, Narotsky DL, Goldsmith J, Weinberg RL, Morgenstern R, et al. Multicenter study of planar technetium 99m pyrophosphate cardiac imaging: Predicting survival for patients with ATTR cardiac amyloidosis. JAMA Cardiol. 2016;1:880-9.

13. Bellevre D, Bailliez A, Delelis F, Blaire T, Agostini D, Mouqet F, et al. Quantitation of myocardial 99mTc-HMDP uptake with new SPECT/CT cadmium zinc telluride (CZT) camera in patients with transthyretin-related cardiac amyloidosis: Ready for clinical use? J Nucl Cardiol. 2020. https://doi.org/10.1007/s12350-020-02274-2.

14. Manrique A, Dudoignon D, Brun S, N'Ganoa C, Cassol E, Legallois D, et al. Quantification of myocardial $(99 \mathrm{~m}) \mathrm{Tc}$-labeled bisphosphonate uptake with cadmium zinc telluride camera in patients with transthyretin-related cardiac amyloidosis. EJNMMI Res. 2019;9:117.

15. Flaherty KR, Morgenstern R, Pozniakoff T, DeLuca A, Castano A, Maurer MS, et al. (99m)Technetium pyrophosphate scintigraphy with cadmium zinc telluride cameras is a highly sensitive and specific imaging modality to diagnose transthyretin cardiac amyloidosis. J Nucl Cardiol. 2020;27:371-80.

16. Perugini E, Guidalotti PL, Salvi F, Cooke RM, Pettinato C, Riva $\mathrm{L}$, et al. Noninvasive etiologic diagnosis of cardiac amyloidosis using 99mTc-3,3-diphosphono-1,2-propanodicarboxylic acid scintigraphy. J Am Coll Cardiol. 2005;46:1076-84.

17. Hutt DF, Fontana M, Burniston M, Quigley AM, Petrie A, Ross JC, et al. Prognostic utility of the Perugini grading of $99 \mathrm{mTc}-\mathrm{DPD}$ scintigraphy in transthyretin (ATTR) amyloidosis and its relationship with skeletal muscle and soft tissue amyloid. Eur Heart J Cardiovasc Imaging. 2017;18:1344-50.

18. Maurer MS, Sultan MB, Rapezzi C. Tafamidis for transthyretin amyloid cardiomyopathy. N Engl J Med. 2019;380:196-7.

19. Dorbala S, Vangala D, Semer J, Strader C, Bruyere JR Jr, Di Carli MF, et al. Imaging cardiac amyloidosis: A pilot study using (1)(8)F-florbetapir positron emission tomography. Eur J Nucl Med Mol Imaging. 2014;41:1652-62.

Publisher's Note Springer Nature remains neutral with regard to jurisdictional claims in published maps and institutional affiliations. 